\title{
Roscovitine ameliorates endotoxin-induced uveitis through neutrophil apoptosis
}

\author{
ZHAO-XIN JIANG ${ }^{*}$, SUO QIU* ${ }^{*}$, BING-SHENG LOU, YAO YANG, WEN-CONG WANG and XIAO-FENG LIN \\ State Key Laboratory of Ophthalmology, Zhongshan Ophthalmic Center, \\ Sun Yat-Sen University, Guangzhou, Guangdong 510060, P.R. China
}

Received August 11, 2015; Accepted May 9, 2016

DOI: $10.3892 / \mathrm{mmr} .2016 .5362$

\begin{abstract}
Neutrophils have been recognized as critical response cells during the pathogenesis of endotoxin-induced uveitis (EIU). Apoptosis of neutrophils induced by roscovitine has previously been demonstrated to ameliorate inflammation in several in vivo models. The present study aimed to assess whether roscovitine ameliorates EIU. EIU was induced in female C57BL/6 mice by a single intravitreal injection of lipopolysaccharide (LPS; $250 \mathrm{ng}$ ). The mice were divided into three groups as follows: LPS alone, LPS plus vehicle, LPS plus roscovitine $(50 \mathrm{mg} / \mathrm{kg})$. The mice were euthanized 12, 24, 48 and $72 \mathrm{~h}$ after LPS-induced uveitis. Accumulation of inflammatory cells in the vitreous body was confirmed by immunohistochemistry, and quantified following hematoxylin and eosin staining. Terminal deoxynucleotidyl transferase dUTP nick-end labeling was performed to detect of apoptotic cells. The mRNA levels of inflammatory cytokines were analyzed by reverse transcription-quantitative polymerase chain reaction and the changes in protein levels were analyzed by western blotting. Inflammatory cells accumulated in the vitreous near the optic nerve head and the quantity peaked at $24 \mathrm{~h}$ after LPS injection. Immunohistochemistry revealed that the majority of the inflammatory cells were neutrophils. The number of infiltrating cells was similar in the LPS and LPS plus vehicle groups, while there were significantly less in the roscovitine group at $24 \mathrm{~h}$. Apoptosis of neutrophils was observed between 12 and $48 \mathrm{~h}$ after roscovitine injection, while no apoptosis was observed in the other groups. The mRNA expression levels of GMCSF, CINC-1 and ICAM-I peaked at $12 \mathrm{~h}$ after LPS injection, and decreased to normal levels at $72 \mathrm{~h}$. This trend in mRNA expression was similar in
\end{abstract}

Correspondence to: Professor Xiao-Feng Lin, State Key Laboratory of Ophthalmology, Zhongshan Ophthalmic Center, Sun Yat-sen University, 54 Xianlie Southern Street, Guangzhou, Guangdong 510060, P.R. China

E-mail: linxiaof@mail.sysu.edu.cn

*Contributed equally

Key words: endotoxin, uveitis, roscovitine, neutrophil, apoptosis the LPS and LPS plus vehicle groups; however, the expression levels decreased more quickly in the roscovitine group at 24 and 48 h. Following roscovitine administration, upregulated cleaved caspase 3 expression levels and downregulated Mcl-1 expression levels were observed. In conclusion, roscovitine ameliorates EIU by effecting neutrophil apoptosis. Timely apoptosis of neutrophils may be an effective process to promote the amelioration of EIU.

\section{Introduction}

Uveitis is one of the most harmful ocular conditions and can affect any part of the eye. In developed countries, uveitis affects $\sim 200 / 100,000$ people in the general population, and $35 \%$ of patients suffer severe visual impairment $(1,2)$. Endotoxin-induced uveitis (EIU) is an animal model of human uveitis induced by lipopolysaccharide (LPS), a component of the outer membranes of gram-negative bacteria $(3,4)$. A major pathological characteristic of EIU is the ocular infiltration of inflammatory cells (5).

The polymorphonuclear leucocyte, or neutrophil, has long been recognized as an early and key response cell in the pathogenesis of EIU $(6,7)$. Through the production of proteolytic enzymes and toxic intermediates, neutrophils are essential for innate immunity (8). However, these defense processes, which occur through destroying and digesting extraneous matter, are potentially deleterious to tissues (9). Thus, it is vital that neutrophils are cleared in a timely manner following achieving their physiological function $(10,11)$. As the removal of neutrophils relies on apoptosis or necrosis, it is critical that the neutrophils undergo apoptosis to maintain the intact plasma membrane (12).

Roscovitine, also termed Seliciclib or CYC202, is a selective cyclin-dependent kinase inhibitor (CDKi) originally designed to suppress tumor cell growth and division (13-15). Notably, this ability to inhibit the cell cycle and induce apoptosis, particularly in polymorphonuclear leukocytes, subsequently improved the resolution of inflammation (16). Its pro-resolution has been demonstrated in several in vivo models, including pleurisy, arthritis and meningitis $(16,17)$.

Therefore, the present study aimed to assess whether roscovitine promoted the apoptosis of neutrophils and subsequently attenuated the inflammatory response in an EIU mouse model. 


\section{Materials and methods}

Animals. Female C57BL/6 mice (age, 6-8 weeks; weight, 18-20 g) were purchased from Southern Medical University (Guangzhou, China). All the animal procedures were performed according to the Association for Research in Vision and Ophthalmology Statement for the Use of Animals in Ophthalmic and Vision Research. The protocol was approved by the Animal Care and Use Committee of the Zhongshan Ophthalmic Center of Sun Yat-Sen University (Guangzhou, China; ethics approval no. 2014-055).

The mice were housed in a temperature controlled environment $\left(22 \pm 1^{\circ} \mathrm{C}\right.$; humidity, $\left.55 \pm 5^{\circ} \mathrm{C}\right)$ with a 12 -h light/dark cycle and access to food and water ad libitum. The mice were divided into three groups: i) LPS group, in which mice were intravitreally injected with LPS; ii) LPS plus vehicle group, in which mice were intravitreally injected with LPS and subsequently treated with the vehicle of roscovitine; iii) roscovitine group, in which mice were intravitreally injected with LPS and subsequently treated with roscovitine. Age-matched untreated mice were used as controls.

Induction of EIU. To induce EIU, each mouse eye received a single intravitreal injection of $1 \mu 1$ sterile pyrogen-free saline, containing 250 ng E. coli 055:B5 endotoxin (Sigma-Aldrich, St. Louis, MO, USA).

The injection was performed, according to a previous study (18). Briefly, the pupils were dilated by the instillation of one drop of 5\% tropicamide (Santen Pharmaceutical Co., Ltd., Osaka, Japan). One drop of $1 \%$ tetracaine was administered for local anesthesia. Intravitreal injections $(1 \mu \mathrm{l})$ were administered in the right eye using sterile syringes fitted with a 30-gauge needle (Hamilton Bonaduz AG, Bonaduz, Switzerland).

Administration of roscovitine. The mice received a single intraperitoneal injection of $50 \mathrm{mg} / \mathrm{kg}$ of roscovitine $10 \mathrm{~min}$ after the intravitreal injection of LPS. The dose of $50 \mathrm{mg} / \mathrm{kg}$ roscovitine is safe and a high dose of $2 \mathrm{~g} / \mathrm{kg}$ is well-tolerated $(19,20)$.

Roscovitine was purchased from Selleck Chemicals (Houston, TX, USA). The solution was made, according to a previous study (21). Briefly, roscovitine was initially dissolved in dimethyl sulfoxide (1 volume). A carrier solution was produced from a diluent containing $10 \%$ Tween 80 (Guanghua Sci-Tech Co., Ltd., Guangzhou, China), 20\% N, N-dimethylacetamide (Guanghua Sci-Tech Co., Ltd.) and 70\% polyethylene glycol 400 (Guanghua Sci-Tech Co., Ltd.).

Histopathology. The mice were sacrificed by cervical dislocation at 12, 24, 48 and $72 \mathrm{~h}$ after LPS-induced uveitis. The eyes were enucleated and fixed in $4 \%$ paraformaldehyde at room temperature overnight. The eyeballs were embedded in paraffin. Serial 5- $\mu \mathrm{m}$ sections were cut through the cornea-optic nerve axis. Paraffin-embedded serial sections were deparaffinized in xylene, rehydrated through a graded ethanol series, and washed with phosphate-buffered saline (PBS). The tissue sections were stained by hematoxylin and eosin (Shanghai Huntz Enterprises Co., Ltd., Shanghai, China). The number of stained cells in the vitreous body near the optic nerve head were quantified under light microscopy ( $\mathrm{n}=6$ per group).
Immunohistochemistry. The mice were sacrificed by cervical dislocation at 12, 24, 48 and $72 \mathrm{~h}$ after LPS-induced uveitis. The enucleated eyes were fixed with $4 \%$ paraformaldehyde, embedded in paraffin, cut into 5- $\mu \mathrm{m}$ serial sections and deparaffinized. Antigen retrieval was performed by heating sections in a microwave oven in citrate buffer $[0.01 \mathrm{~mol} / \mathrm{l}(\mathrm{pH}$ 6.0)] for $30 \mathrm{~min}$. Endogenous peroxidase was eliminated by incubation in $3 \%$ hydrogen peroxide in PBS for $10 \mathrm{~min}$, and the tissue sections were washed in PBS for 5 min three times, and incubated in $10 \%$ goat serum (Biosharp, Hefei, China) for $2 \mathrm{~h}$. The tissue sections were incubated with rabbit polyclonal antibodies against cluster of differentiation 177 (CD177; dilution, 1:100; Abcam, Cambridge, UK; cat. no. ab203025) overnight at $4^{\circ} \mathrm{C}$, followed by a goat anti-rabbit horseradish peroxidase (HRP)-conjugated secondary antibody (dilution, 1:1,000; Abcam; cat. no. ab97200) for $1 \mathrm{~h}$ at $37^{\circ} \mathrm{C}$ and stained with peroxidase. The stained cells in the vitreous body near the optic nerve head were observed under light microscopy ( $\mathrm{n}=6$ per group).

Terminal deoxynucleotidyltransferasedUTP nick-end labeling (TUNEL). TUNEL was performed for the in situ detection of apoptotic cells using the tetramethylrhodamine-red-based in situ Death Detection kit from Roche Diagnostics (Roche Diagnostics, Shanghai, China). The mice were euthanized 12, 24, 48 and $72 \mathrm{~h}$ after LPS-induced uveitis. Following antigen retrieval, as described for immunohistochemistry, the paraffin-embedded serial sections were incubated with the TUNEL reaction mixture for $1 \mathrm{~h}$ at $37^{\circ} \mathrm{C}$ in a humidified atmosphere in the dark. TUNEL-positive cells were visualized with a fluorescence microscope ( $\mathrm{n}=6$ per group).

Reverse transcription-quantitative polymerase chain reaction $(R T-q P C R)$ analysis of the inflammatory cytokines. Total RNA was extracted from the freshly enucleated eyeball using TRIzol reagent (Invitrogen; Thermo Fisher Scientific, Inc. Waltham, MA, USA), according to the manufacturer's instructions. cDNA was generated using the PrimeScript RT reagent kit (Takara Biotechnology Co., Ltd., Dalian, China) at $37^{\circ} \mathrm{C}$ for $15 \mathrm{~min}$, followed by $85^{\circ} \mathrm{C}$ for $5 \mathrm{sec}$ and then maintained at $4^{\circ} \mathrm{C}$. RT-qPCR was performed according to the manufacturer's instructions using a Bio-Rad C-1000 Thermal Cycler (Bio-Rad Laboratories, Inc., Hercules, CA, USA). The primer sequences are listed in Table I. All primers were purchased from Invitrogen (Thermo Fisher Scientific, Inc.).

RT-qPCR was performed in a $20 \mu 1$ reaction mixture using the FastStart Universal SYBR-Green Master reagent (Roche Diagnostics) on an ABI PRISM 7000 sequence detection system (Applied Biosystems; Thermo Fisher Scientific, Inc.). The PCR conditions were as follows: 1 cycle at $95^{\circ} \mathrm{C}$ for $10 \mathrm{~min}$, followed by 40 cycles at $95^{\circ} \mathrm{C}$ for $15 \mathrm{sec}$ and $60^{\circ} \mathrm{C}$ for $1 \mathrm{~min}$. The $2^{-\Delta \Delta \mathrm{Cq}}$ method was used to estimate the relative transcript levels (22). The levels of GAPDH amplification were used to normalize each sample $\mathrm{Cq}$ value. Units are expressed as relative quantification ( $\mathrm{n}=6$ per group).

Western blotting. Freshly enucleated eyeballs were homogenized and solubilized in a radioimmunoprecipitation assay lysis buffer containing 1\% phenylmethylsulfonyl fluoride (Biocolors, Shanghai, China). The total protein concentration was determined using a bicinchoninic acid protein assay kit 
Table I. Sequences of primers for reverse transcription-polymerase chain reaction.

\begin{tabular}{lll}
\hline Gene & \multicolumn{1}{c}{ Forward } & \multicolumn{1}{c}{ Reverse } \\
\hline IL-1 & 5'-GAAATGCCACCTTTTGACAGTG-3' & 5'-TGGATGCTCTCATCAGGACAG-3' \\
GMCSF & 5'-TGGAAGCATGTAGAGGCCATCA-3' & 5'-GCGCCCTTGAGTTTGGTGAAAT-3' \\
CINC-1 & 5'-GTCATAGCCACACTCAAG-3' & 5'-CCATCAGAGCAGTCTGTC-3' \\
ICAM-1 & 5'-AGATGACCTGCAGACGGAAG-3' & 5'-GGCTGAGGGTAAATGCTGTC-3' \\
GAPDH & 5'-AGGTCGGTGTGAACGGATTTG-3' & 5'-TGTAGACCATGTAGTTGAGGTCA-3'
\end{tabular}

IL-1, interleukin-1; GMCSF, granulocyte-macrophage colony-stimulating factor; CINC-1, cytokine-induced neutrophil chemoattractant 1; ICAM-1, intercellular adhesion molecule 1; GAPDH, glyceraldehyde-3-phosphate dehydrogenase.
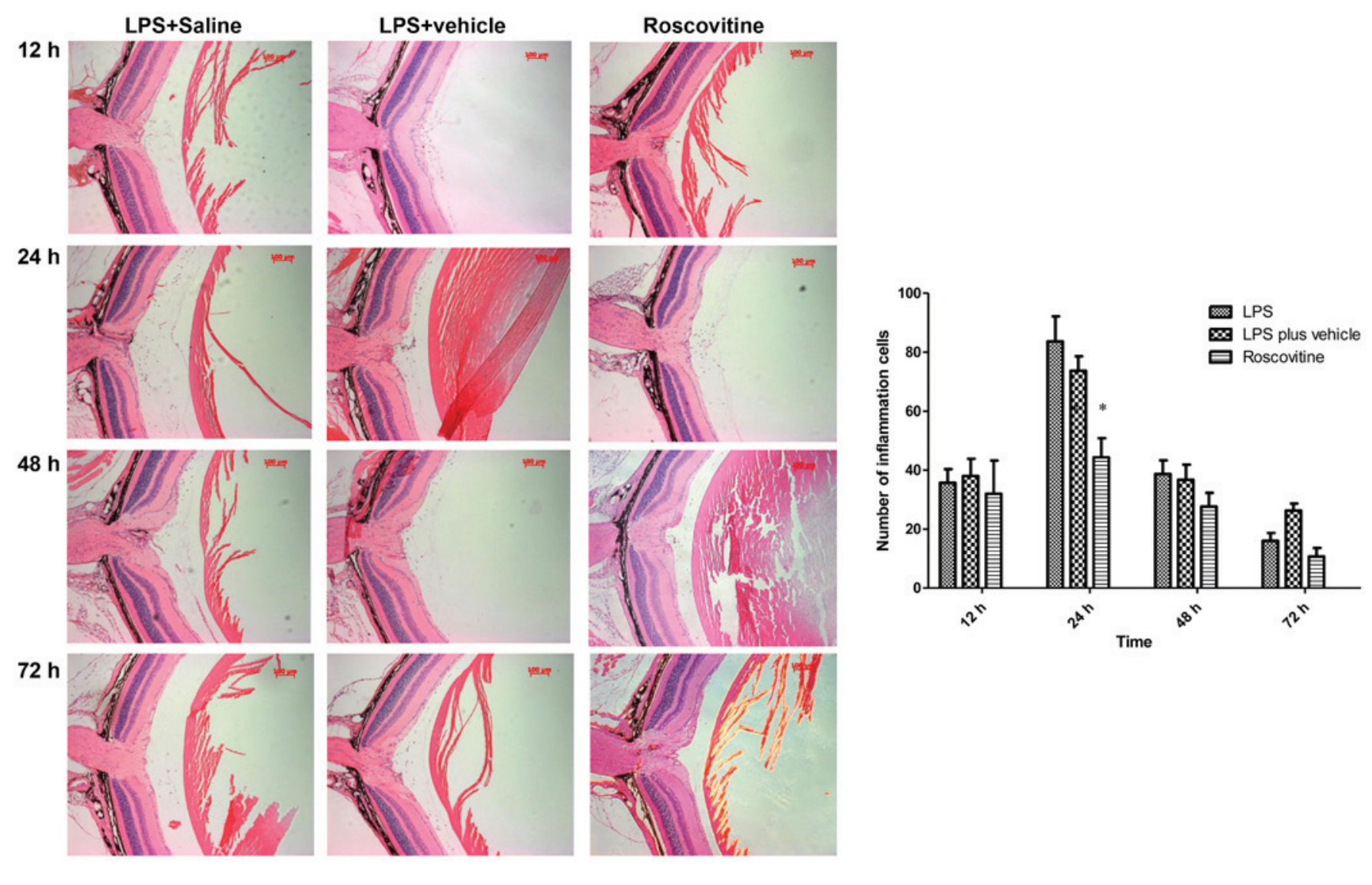

Figure 1. Hematoxylin and eosin-stained evaluation of inflammatory cells in the vitreous near the optic nerve. The quantity of inflammatory cells increased, peaked at $24 \mathrm{~h}$ after LPS injection, and subsequently decreased gradually to normal levels at $72 \mathrm{~h}$ in the three groups. In the roscovitine group cell numbers were lower at the respective time points, the difference was significant at $24 \mathrm{~h}$. "P<0.05 vs. the LPS group. Magnification, $\mathrm{x} 100, \mathrm{scale}$ bar=100 $\mu \mathrm{m}$. LPS, lipopolysaccharide.

(Cwbiotech, Beijing, China), and detected using a microplate reader (BioTek Instruments, Winooski, USA). A total of $15 \mu \mathrm{g}$ protein lysate was resolved on 10, 12 or $15 \%$ SDS-PAGE gels (MDBio, Qingdao, China), according to the molecular weight of the target protein, and was subsequently transferred onto nitrocellulose membranes (EMD Millipore, Billerica, MA, USA). The blots were blocked with $5 \%$ bovine serum albumin (Sigma-Aldrich) and probed overnight at $4^{\circ} \mathrm{C}$ with primary antibodies as follows: Rabbit polyclonal cleaved caspase 3 (dilution, 1:1,000; Cell Signaling Technology, Inc., Danvers, MA, USA; cat. no. 9661), rabbit polyclonal cyclooxygenase 2 (COX-2; cat. no. ab15191), rabbit polyclonal phosphorylated

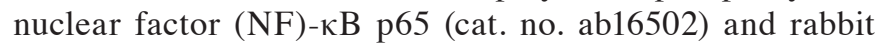

monoclonal myeloid cell leukemia 1 (Mcl-1; cat. no. ab32087), all diluted at 1:1,000 and obtained from Abcam. The blots were washed with Tris-buffered saline with Tween-20 and incubated with a goat anti-rabbit HRP-conjugated secondary antibody (dilution, 1:1,000; cat. no. ab6721) for $2 \mathrm{~h}$ at $37^{\circ} \mathrm{C}$. The blots were developed using Immobilon ${ }^{\mathrm{TM}}$ Western Chemiluminescent HRP Substrate (EMD Millipore). The bands were analyzed using Image $\mathbf{J}$ software (Version 1.43; National Institutes of Health, Bethesda, MD, USA). The measurements were repeated in triplicate for each experiment ( $\mathrm{n}=6$ per group). To ascertain that the blots were loaded with equal quantities of proteins, they were also incubated with an antibody against $\beta$-actin (1:1,000; Abcam; cat. no. ab8229). 


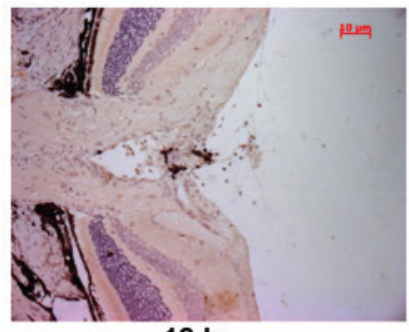

$12 \mathrm{~h}$

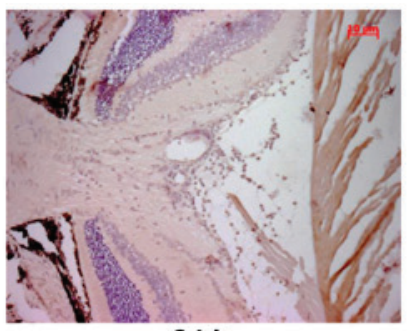

$24 \mathrm{~h}$

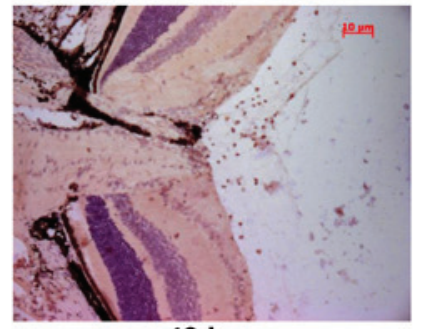

$48 \mathrm{~h}$

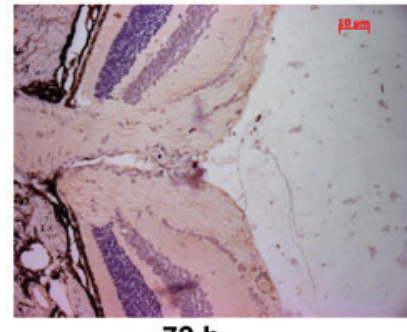

$72 \mathrm{~h}$

Figure 2. Immunohistochemistry of inflammatory cells in the vitreous body near the optic nerve. The inflammatory cells were labeled with CD177, indicating that the majority of cells in the vitreous were neutrophils. Magnification, x200, scale bar=10 $\mu \mathrm{m}$.

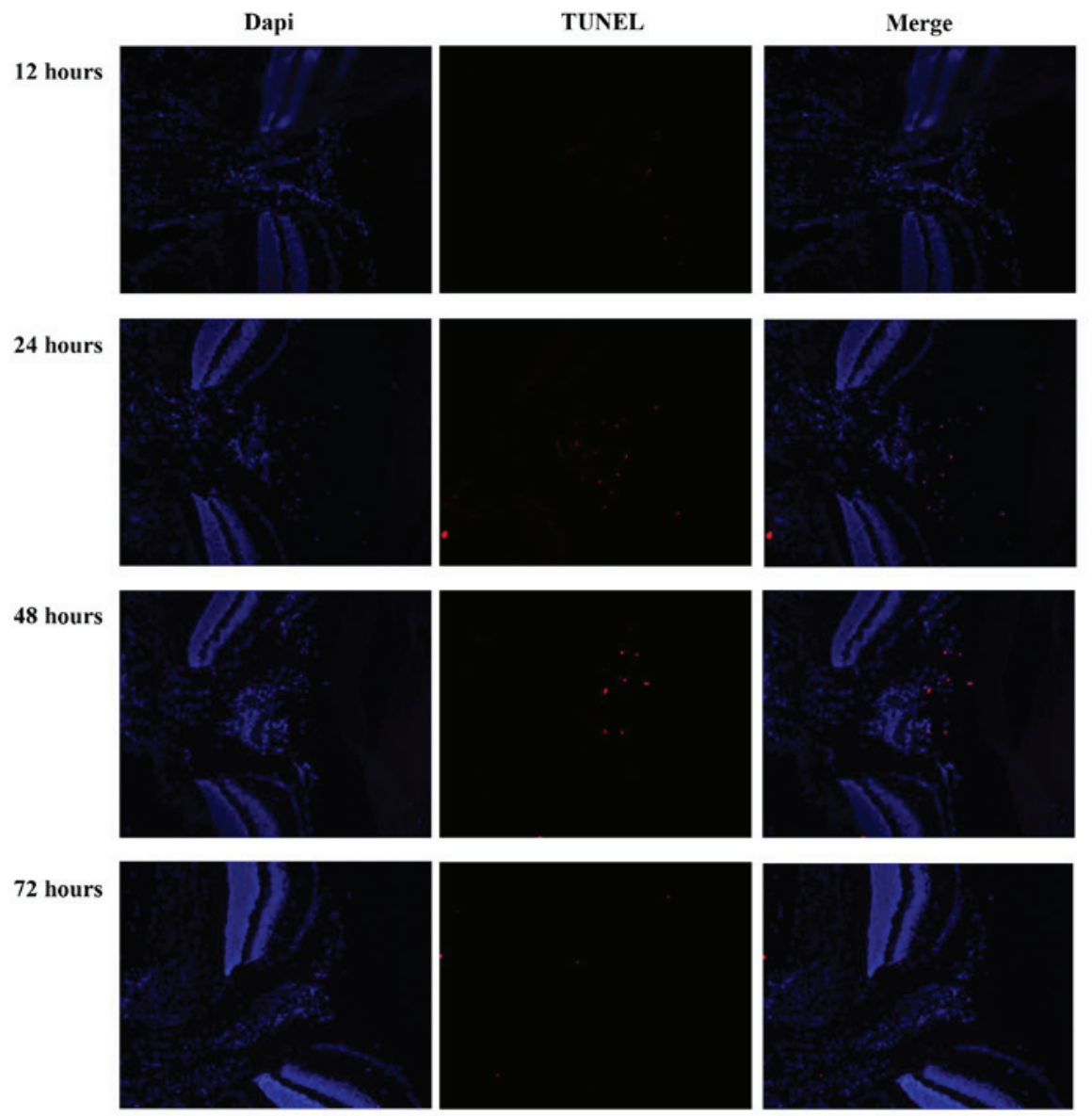

Figure 3. Terminal deoxynucleotidyl transferase dUTP nick-end labeling analysis measured the apoptosis of neutrophils following the administration of roscovitine. Apoptosis occurred at 12, 24 and $48 \mathrm{~h}$, but no marked detection was observed at $72 \mathrm{~h}$ after roscovitine injection. Apoptosis was limited in the inflammatory cells in the vitreous body. Magnification, x200.

Statistical analysis. Data was analyzed using GraphPad Prism (version 6.0; GraphPad Software, Inc., La Jolla, CA, USA). All values are expressed as the mean \pm standard deviation. The results were analyzed by one-way analysis of variance followed by a Bonferroni post-hoc test for multiple comparisons. $\mathrm{P}<0.05$ was considered to indicate a statistically significant difference.

\section{Results}

Neutrophils infiltration occurs following LPS injection; however, less accumulation is observed in the roscovitine group. Hematoxylin and eosin staining revealed that the inflammatory cells accumulated in the vitreous body close to the optic nerve head following LPS injection (Fig. 1). In the three groups, the quantity of inflammatory cells increased, peaked at $24 \mathrm{~h}$ after LPS injection, and subsequently decreased gradually to normal levels at $72 \mathrm{~h}$. The cells numbers were similar in the LPS and LPS plus vehicle groups at identical time points, while cell numbers were lower in the roscovitine group at the respective time points. In particular, the number of inflammatory cells was significantly lower in the roscovitine groups compared with the LPS group at $24 \mathrm{~h}$ $(\mathrm{P}=0.037)$. 

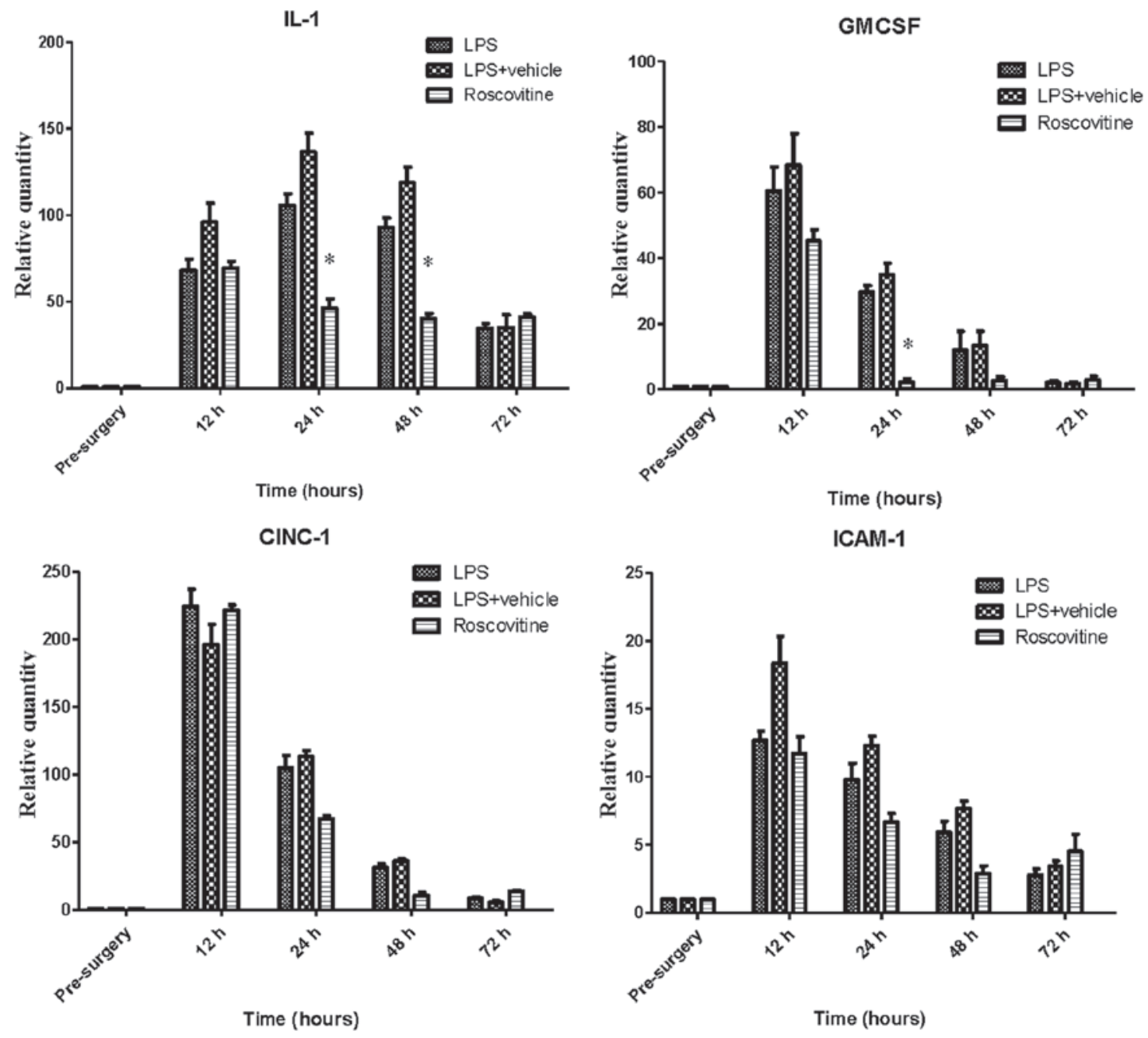

Figure 4. Reverse transcription-quantitative polymerase chain reaction analysis of mRNA expression for inflammatory cytokines. The relative expression level was similar in the LPS and LPS plus vehicle groups at the identical time points, whereas the relative mRNA expression levels were lower in the roscovitine group at the respective time point. The results for IL-1 were significant at 24 and $48 \mathrm{~h}$ when compared with the LPS group ( $\mathrm{P}=0.042$ and 0.033 , respectively). The difference of GMCSF was significant at $24 \mathrm{~h}$ when compared with LPS groups $(\mathrm{P}=0.026)$. " $\mathrm{P}<0.05$ vs. the LPS group. LPS, lipopolysaccharide; IL-1, interleukin-1; GMCSF, granulocyte-macrophage colony-stimulating factor; CINC-1, cytokine-induced neutrophil chemoattractant 1; ICAM-1, intercellular adhesion molecule 1.

Immunohistochemistry demonstrated that the infiltrating inflammatory cells were labeled with CD177, indicating that the majority of cells in the vitreous were neutrophils (Fig. 2).

Apoptosis of neutrophils is observed following the administration of roscovitine. The TUNEL assay demonstrated that apoptosis occurred at 12, 24 and 48 h; however, no significant detection was observed at $72 \mathrm{~h}$ after roscovitine injection (Fig. 3). Apoptosis was observed in the inflammatory cells in the vitreous body only, and no apoptosis was observed in the retinal cells. No apoptosis was observed in untreated or other groups at the $24 \mathrm{~h}$ time-point after LPS injection.

Inflammatory response decreases following administration of roscovitine. RT-qPCR analysis demonstrated that the mRNA expression level of interleukin 1 increased, peaked at $24 \mathrm{~h}$ after LPS injection, and decreased gradually by $72 \mathrm{~h}$. The mRNA expression levels of granulocyte-macrophage colony-stimulating factor $(G M C S F)$, cytokine-induced neutrophil chemoattractant $1(C I N C-1)$ and intercellular adhesion molecule $1(I C A M-1)$ peaked at $12 \mathrm{~h}$, then decreased rapidly to the normal levels (Fig. 4). The relative mRNA levels were similar in the LPS and LPS plus vehicle groups at the identical time points, while the relative levels were lower in the roscovitine group at the respective time points. In particular, the mRNA levels of interleukin 1 in the roscovitine groups were significantly reduced compared with the LPS group at 24 $(\mathrm{P}=0.042)$ and $48 \mathrm{~h}(\mathrm{P}=0.033)$.

Western blot analysis revealed that the protein expression level of COX-2 was markedly increased at 12 and $24 \mathrm{~h}$; however, the level decreased to almost normal levels at 48 and $72 \mathrm{~h}$ in the four groups (Fig. 5). The expression of COX-2 was significantly lower in the roscovitine group compared with the control groups at $24(\mathrm{P}=0.021)$ and $72 \mathrm{~h}(\mathrm{P}=0.035)$. A similar trend was observed in the levels of phosphorylated $\mathrm{NF}-\kappa \mathrm{B}$ p 65, and compared with the LPS group, significantly less expression was observed in the roscovitine group at $48 \mathrm{~h}$ $(\mathrm{P}=0.047)$.

Roscovitine promotes apoptosis upregulating the caspase 3 and downregulating the Mcl-1 expression levels. Western blot analysis demonstrated that the expression of cleaved caspase 3 

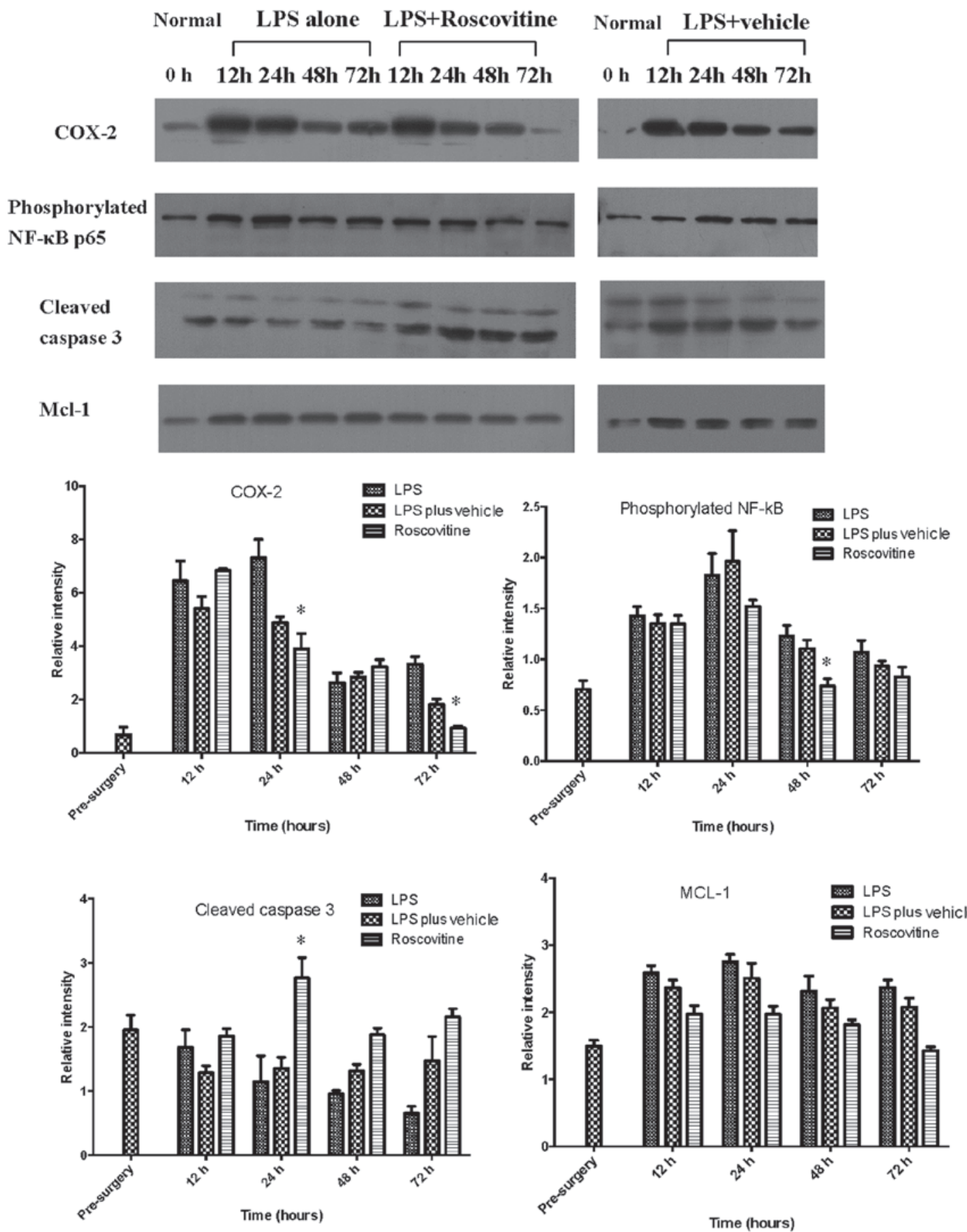

Figure 5. Roscvovitine promotes apoptosis through increased expression of cleaved caspase 3 and decreased expression of Mcl-1. The expression of COX-2, phosphorylated NF-kB and Mcl-1 were increased at $24 \mathrm{~h}$, but decreased gradually over time. The expression of COX-2 was significantly different between the LPS and roscovitine groups at 24 and $72 \mathrm{~h}(\mathrm{P}=0.021$ and 0.035 , respectively). The expression of phosphorylated NF- $\mathrm{kB}$ was significantly decreased at $48 \mathrm{~h}(\mathrm{P}=0.047)$. The expression of cleaved caspase 3 was markedly increased in the roscovitine group at $24 \mathrm{~h}(\mathrm{P}=0.029)$. No statistically difference was observed in Mcl-1 expression among the groups. "P $<0.05$ vs. the LPS group. LPS, lipopolysaccharide; COX-2, cyclooxygenase-2; NF- $\mathrm{BB}$, nuclear factor- $\mathrm{kB}$; Mcl-1, myeloid cell leukemia 1.

was markedly increased in the roscovitine group at $24 \mathrm{~h}$ compared with the untreated group $(\mathrm{P}=0.029)$, whereas it was maintained at similar levels at other time points (Fig. 5). The expression level of Mcl-1 increased and was maintained at a stable level at different time points following LPS injection, compared with the untreated group. In addition, the Mcl-1 expression of the roscovitine group was higher compared with the untreated group; however, it was lower than other groups.
No statistically significant difference was observed in Mcl-1 expression levels among the groups.

\section{Discussion}

Polymorphonuclear leucocytes are the predominant infiltrating cell in EIU. In the present study, a selective CDKi was applied as a potential drug and the results demonstrated that resolution 
of inflammation was accelerated through the increased apoptosis of neutrophils.

The CDKs are part of a family of serine/threonine protein kinases, and their ability to regulate the cell cycle has made them an attractive target for anticancer therapies (13). The preliminary research was initiated in marine starfish oocytes in the late $1970 \mathrm{~s}$, and $21 \mathrm{CDK}$ genes were discovered in the human genome. Of these, 11 are considered as classical CDKs (15,23).

Roscovitine is a 2,6,9-trisubstituted purine first synthesized in 1997. It is a selective inhibitor of CDK 1, 2, 5, 7 and 9 isoforms, and is capable of actively promoting apoptosis (23). In multiple myeloma cells, MacCallum et al (13) reported that roscovitine induces cell death by inhibition of RNA polymerase II-dependent transcription and downregulation of Mcl-1. Rossi et al (16) identified that roscovitine treatment results in caspase 3 cleavage. Additionally, Rossi et al demonstrated that roscovitine abrogates the antiapoptotic effects of important survival factors, including dibutyryl-cAMP, GMCSF and LPS (16). These survival factors utilize the major inflammatory signaling pathways, phosphoinositide 3-kinase, $\mathrm{NF}-\kappa \mathrm{B}$, Janus kinase/signal transducers and activators of transcription and mitogen-activated protein kinases, to augment neutrophil survival (24).

However, neutrophils are terminally differentiated cells and remain in the $G_{0}$ phase, therefore, roscovitine may be considered to have no effect (25). Notably, previous in vitro analysis demonstrated that human neutrophils express CDK1, CDK2 and CDK5 (16). Furthermore, although no change in the protein expression of CDK1 or CDK2 was demonstrated, changes in the function of $\mathrm{CDK}$ and expression of their binding partner cyclins were observed $(16,26)$. Rossi et al (16) demonstrated a prompt reduction in CDK1 activity during induction of apoptosis by the activating Fas antibody, CH11. Once apoptosis has been engaged, the neutrophil secretory activity is sequestered; the cells remain intact and are phagocytosed by macrophages (27). Phagocytosis of apoptotic cells stimulates macrophages to polarize into an M2 or pro-resolution phenotype (28).

EIU in mice results in a prominent posterior vitritis and an accumulation of inflammatory cells in the vitreous near the optic nerve head (29). Previous histological studies have revealed an infiltration of leucocytes between 4 and $6 \mathrm{~h}$ after LPS injection, and cell numbers increasing to a maximum at $24 \mathrm{~h} \mathrm{(30).} \mathrm{The} \mathrm{neutrophil} \mathrm{is} \mathrm{consistently} \mathrm{the} \mathrm{first} \mathrm{cell} \mathrm{type}$ to arrive at a site of inflammation, to phagocytose a variety of foreign particles. Neutrophils also produce chemokines, including CINC-1, to attract more inflammatory cells, and express adhesion molecules, including ICAM-1, to facilitate cell migration $(31,32)$

In the present study, neutrophils predominantly accumulated in the vitreous near the optic nerve head, as demonstrated by histological analysis, and their quantity peaked at $24 \mathrm{~h}$ after LPS intravitreal injection. Additionally, apoptosis was observed in the TUNEL analysis, and the downregulated protein expression levels of Mcl-1 and upregulated expression of cleaved caspase- 3 were observed following the administration of roscovitine. Following the apoptosis of neutrophils, lower mRNA expression of $I L-I$ and $\mathrm{NF}-\kappa \mathrm{B}$ p 65 phosphorylation were observed, indicating that roscovitine promotes inflammatory resolution. Furthermore, lower mRNA expression levels of GMCSF, CINC-1 and $I C A M-1$ were observed following roscovitine treatment, which assists in explaining the lower quantity of neutrophils in the vitreous of the roscovitine group. Thus, the administration of roscovitine promoted the apoptosis of neutrophils accumulated in the vitreous following LPS stimulation, and the increased apoptosis of neutrophils accelerated the resolution of EIU.

Suppressed neutrophil apoptosis has been detected in patients with inflammatory diseases, including pneumonia, sepsis and rheumatoid arthritis, where enhanced neutrophil apoptosis ameliorates the prognosis. The study by Rossi et al (16) reported that roscovitine markedly enhanced the resolution of established neutrophil-dependent inflammation in carrageenan-elicited acute pleurisy, bleomycin-induced lung injury and passively-induced arthritis in mice. Koedel et al (17) reported that roscovitine significantly improved the resolution of the inflammation following pneumococcal infection and accelerated the recovery. A previous study by Hoogendijk et al (33) reported that roscovitine reduced lung inflammation induced by lipoteichoic acid and Streptococcus pneumonia.

In conclusion, roscovitine ameliorated EIU by altering neutrophil apoptosis. Timely apoptosis of neutrophils may be an effective process to promote the resolution of EIU.

\section{Acknowledgments}

This study was supported by Science and Technology Planning Project of Guangdong Province, China (grant no. 2014A020212165, 2014A020212099).

\section{References}

1. Rathinam SR: Treating uveitis in the developing world setting. Int Ophthalmol Clin 50: 219-228, 2010.

2. Tomkins-Netzer O, Talat L, Bar A, Lula A, Taylor SR, Joshi L and Lightman S: Long-term clinical outcome and causes of vision loss in patients with uveitis. Ophthalmology 121: 2387-2392, 2014.

3. Rosenbaum JT, McDevitt HO, Guss RB and Egbert PR: Endotoxin-induced uveitis in rats as a model for human disease. Nature 286: 611-613, 1980.

4. Mérida S, Palacios E, Navea A and Bosch-Morell F: Macrophages and uveitis in experimental animal models. Mediators Inflamm 2015: 671417, 2015.

5. Metrikin DC, Wilson CA, Berkowitz BA,Lam MK, Wood GK and Peshock RM: Measurement of blood-retinal barrier breakdown in endotoxin-induced endophthalmitis. Invest Ophthalmol Vis Sci 36: 1361-1370, 1995

6. McMenamin PG and Crewe J: Endotoxin-induced uveitis. Kinetics and phenotype of the inflammatory cell infiltrate and the response of the resident tissue macrophages and dendritic cells in the iris and ciliary body. Invest Ophthalmol Vis Sci 36: 1949-1959, 1995

7. Mashimo H, Ohguro N, Nomura S, Hashida N, Nakai K and Tano Y: Neutrophil chemotaxis and local expression of interleukin-10 in the tolerance of endotoxin-induced uveitis. Invest Ophthalmol Vis Sci 49: 5450-5457, 2008.

8. Bennouna S, Bliss SK, Curiel TJ and Denkers EY: Cross-talk in the innate immune system: Neutrophils instruct recruitment and activation of dendritic cells during microbial infection. J Immunol 171: 6052-6058, 2003.

9. Nathan C and Ding A: Nonresolving inflammation. Cell 140: 871-882, 2010.

10. Savill J, Dransfield I, Gregory C and Haslett C: A blast from the past: Clearance of apoptotic cells regulates immune responses. Nat Rev Immunol 2: 965-975, 2002. 
11. Gilroy DW, Lawrence T, Perretti M and Rossi AG: Inflammatory resolution: New opportunities for drug discovery. Nat Rev Drug Discov 3: 401-416, 2004

12. El Kebir D and Filep JG: Role of neutrophil apoptosis in the resolution of inflammation. ScientificWorldJournal 10: 1731-1748 2010.

13. MacCallum DE, Melville J, Frame S, Watt K, Anderson S, Gianella-Borradori A, Lane DP and Green SR: Seliciclib (CYC202, R-Roscovitine) induces cell death in multiple myeloma cells by inhibition of RNA polymerase II-dependent transcription and down-regulation of Mcl-1. Cancer Res 65: 5399-5407, 2005.

14. Bach S, Knockaert M, Reinhardt J, Lozach O, Schmitt S, Baratte B, Koken M, Coburn SP, Tang L, Jiang T, et al: Roscovitine targets, protein kinases and pyridoxal kinase. J Biol Chem 280: 31208-31219, 2005.

15. Cicenas J, Kalyan K, Sorokinas A, Stankunas E, Levy J1, Meskinyte I, Stankevicius V, Kaupinis A and Valius M: Roscovitine in cancer and other diseases. Ann Transl Med 3: 135, 2015

16. Rossi AG, Sawatzky DA, Walker A, Ward C, Sheldrake TA, Riley NA, Caldicott A, Martinez-Losa M, Walker TR, Duffin R, et al: Cyclin-dependent kinase inhibitors enhance the resolution of inflammation by promoting inflammatory cel apoptosis. Nat Med 12: 1056-1064, 2006.

17. Koedel U, Frankenberg T, Kirschnek S, Obermaier B, Häcker H Paul R and Häcker G: Apoptosis is essential for neutrophil functional shutdown and determines tissue damage in experimental pneumococcal meningitis. PLoS Pathog 5: e1000461, 2009.

18. Yadav UC and Ramana KV: Endotoxin-induced uveitis in rodents. Methods Mol Biol 1031: 155-162, 2013.

19. McClue SJ, Blake D, Clarke R, Cowan A, Cummings L, Fischer PM, MacKenzie M, Melville J, Stewart K, Wang S, et al: In vitro and in vivo antitumor properties of the cyclin dependent kinase inhibitor CYC202 (R-roscovitine). Int J Cancer 102: 463-468, 2002

20. Huber RJ: The cyclin-dependent kinase family in the social amoebozoan Dictyostelium discoideum. Cell Mol Life Sci 71 629-639, 2014.

21. Tirado OM, Mateo-Lozano S and Notario V: Roscovitine is an effective inducer of apoptosis of Ewing's sarcoma family tumor cells in vitro and in vivo. Cancer Res 65: 9320-9327, 2005.
22. Livak KJ and Schmittgen TD: Analysis of relative gene expression data using real-time quantitative PCR and the 2(-Delta Delta C(T)) method. Methods 25: 402-408, 2001

23. Meijer L and Raymond E: Roscovitine and other purines as kinase inhibitors. From starfish oocytes to clinical trials. Acc Chem Res 36: 417-425, 2003.

24. Sabroe I, Jones EC, Usher LR, Whyte MK and Dower SK: Toll-like receptor (TLR) 2 and TLR4 in human peripheral blood granulocytes: A critical role for monocytes in leukocyte lipopolysaccharide responses. J Immunol 168: 4701-4710, 2002.

25. Leitch AE, Haslett C and Rossi AG: Cyclin-dependent kinase inhibitor drugs as potential novel anti-inflammatory and pro-resolution agents. Br J Pharmacol 158: 1004-1016, 2009.

26. Knockaert M, Greengard P and Meijer L: Pharmacological inhibitors of cyclin-dependent kinases. Trends Pharmacol Sci 23: 417-425, 2002.

27. Ariel A, Fredman G, Sun YP, Kantarci A, Van Dyke TE, Luster AD and Serhan CN: Apoptotic neutrophils and T cells sequester chemokines during immune response resolution through modulation of CCR 5 expression. Nat Immunol 7: 1209-1216, 2006.

28. Sica A and Mantovani A: Macrophage plasticity and polarization: In vivo veritas. J Clin Invest 122: 787-795, 2012.

29. Smith JR, Hart PH and Williams KA: Basic pathogenic mechanisms operating in experimental models of acute anterior uveitis. Immunol Cell Biol 76: 497-512, 1998.

30. Planck SR, Becker MD, Crespo S, Choi D, Galster K, Garman KL, Nobiling R and Rosenbaum JT: Characterizing extravascular neutrophil migration in vivo in the iris. Inflammation 31: 105-111, 2008.

31. Guex-Crosier Y, Wittwer AJ and Roberge FG: Intraocular production of a cytokine (CINC) responsible for neutrophil infiltration in endotoxin induced uveitis. Br J Ophthalmol 80: 649-653, 1996

32. Carlos TM and Harlan JM: Leukocyte-endothelial adhesion molecules. Blood 84: 2068-2101, 1994.

33. Hoogendijk AJ, Roelofs JJ, Duitman J, van Lieshout MH, Blok DC, van der Poll T and Wieland CW: R-roscovitine reduces lung inflammation induced by lipoteichoic acid and Streptococcus pneumoniae. Mol Med 18: 1086-1095, 2012. 